\title{
Ruptured Sinus of Valsalva Aneurysm Causing Aorto-Atrial Fistula and Acute Heart Failure: A Rare Encounter
}

\author{
Zafar Ali ${ }^{1}$, Talal Asif ${ }^{2}$, Justin P Sheehy ${ }^{3}$, Dany Jacob ${ }^{3}$, Paramdeep Baweja $^{3}$ \\ ${ }^{1}$ University of Kansas Medical Center, Kansas City, KS, USA \\ 2 John H. Stroger, Jr. Hospital of Cook County, Chicago, IL, USA. \\ ${ }^{3}$ University of Missouri-Kansas City, Kansas City, MO, USA
}

Received: 17/01/2020

Accepted: 03/02/2020

Published: 06/03/2020

How to cite this article: Ali Z, Asif T, Sheehy JP, Jacob D, Baweja P. Ruptured sinus of valsava aneurysm causing aorto-artrial fistula and acute heart failure: a rare encounter. EJCRIM 2020;7: doi:10.12890/2020_001499.

Conflicts of Interests: The Authors declare that there are no competing interests.

This article is licensed under a Commons Attribution Non-Commercial 4.0 License

\section{ABSTRACT}

A ruptured sinus of Valsalva aneurysm as a cause of aorto-atrial fistula is very rare. We present the case of a 53-year-old female who presented with symptoms of acute heart failure and suspicion of an aorto-atrial fistula found on a transthoracic echocardiogram, which was confirmed on transesophageal echocardiography. A coronary angiogram showed normal coronary arteries but confirmed the right aortoatrial fistula on aortogram. She underwent successful surgical repair of the fistula. Her postoperative echocardiogram showed a normal right atrium and right ventricle with no shunt. A ruptured sinus of Valsalva aneurysm is a devastating event and presents as acute heart failure. Prompt diagnosis and surgical repair is necessary to prevent mortality.

\section{LEARNING POINTS}

- A ruptured sinus of Valsalva aneurysm causing an aorto-atrial fistula can present as acute heart failure.

- A transthoracic echocardiogram can identify the presence and location of a sinus of Valsalva aneurysm, while a transesophageal echocardiogram can recognize fistulous tract formation. Cardiac magnetic resonance imaging and computed tomography can confirm or better categorize echocardiographic findings.

- The management of an aorto-atrial fistula is surgical intervention, involving a patch repair.

\section{KEYWORDS}

Sinus of Valsalva aneurysm, aorto-atrial fistula, heart failure

\section{INTRODUCTION}

Sinus of Valsalva aneurysms (SVAs) are rare cardiac anomalies, accounting for $0.09-1.5 \%$ of all structural cardiac abnormalities ${ }^{[1]}$. SVAs may be congenital or acquired and are characterized by expansion of the wall of the aortic root between the aortic valve annulus and the sinotubular junction ${ }^{[2]}$. SVAs are typically silent and diagnosed incidentally by imaging performed for other reasons; however, the rupture of SVAs is a life-threatening event associated with a high mortality ${ }^{[3]}$. SVAs most commonly rupture into the right atrium (29\%) or the right ventricle (60\%), whereas rupture into the left-sided chambers occurs in only $10 \%$ of cases ${ }^{[4]}$. Aorto-atrial fistulas (AAFs) are seen infrequently ${ }^{[5]}$ and have been reported in only a few cases $^{[6,7]}$.

Here we present an exceedingly rare case of a patient with a ruptured SVA from the non-coronary cusp into the right atrium, leading to AAF formation and acute heart failure. 


\section{CASE DESCRIPTION}

A 53-year-old female patient with a past medical history of essential hypertension, treatment-naïve hepatitis $C$ and compensated liver cirrhosis presented with a 3 day history of rapidly progressive dyspnea on exertion. It was associated with new-onset orthopnea, paroxysmal nocturnal dyspnea, and bilateral lower extremity edema.

On presentation, she had blood pressure of $121 / 75 \mathrm{mmHg}$, a heart rate of 72 beats/minute, temperature of $98^{\circ} \mathrm{F}$ and a respiratory rate of 16 breaths/minute, with an oxygen saturation of $96 \%$ on room air. On physical examination, she had elevated jugular venous distension to the mandible, bilateral ankle 2+ pitting edema and a new grade 3/6 holosystolic murmur throughout the precordium, loudest at the right second intercostal space. Pulmonary examination revealed bilateral basal fine inspiratory crackles. The remainder of the examination was unremarkable.

An electrocardiogram showed no acute or chronic ischemic changes. A comprehensive metabolic panel, complete blood count, D-dimer and troponin were normal. Plasma N-terminal pro-brain natriuretic peptide (NT-proBNP) was elevated at 3,800 pg/mL. A chest X-ray demonstrated bilateral interstitial infiltrates consistent with pulmonary edema without cardiomegaly.

She was admitted to the hospital and commenced on intravenous diuretics. A transthoracic echocardiogram (TTE) was performed, which showed an ejection fraction of 60-65\%, a dilated right atrium and right ventricle, with diastolic flattening of the interventricular septum suggestive of volume overload. Furthermore, there was turbulent diastolic color flow, which appeared to originate from the left ventricular outflow tract and flowed into the right atrium.

A transesophageal echocardiogram (TEE) was performed and showed a continuous left-to-right flow from either the left ventricular outflow tract or sinus of Valsalva into the right atrium related to the ruptured SVA. Moreover, diastolic pressure gradients of greater than $50 \mathrm{mmHg}$ above the right atrial pressure were noted, which were more consistent with a diagnosis of ruptured SVA. Given the unclear origin of fistula, a cardiovascular computed tomography (CT) angiogram was performed which showed a connection from the non-coronary cusp into the right atrium related to the sinus of Valsalva rupture into the right atrium (Figs 1 and 2).

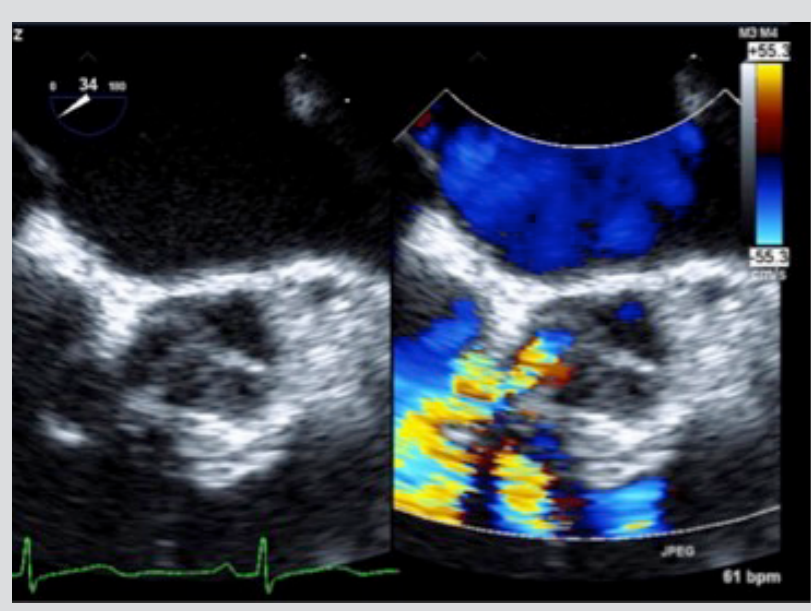

Figure 1. Transesophageal echocardiography in short axis showing the ruptured SVA creating an abnormal connection with the right atrium



Figure 2. Computer Tomography of the chest with contrast showing the ruptured SVA creating an abnormal connection with the right atrium

Due to the patient's acute heart failure, a cardiothoracic surgery consultation was held and immediate surgical intervention planned. A coronary angiogram was then undertaken as part of the preoperative evaluation, which showed normal coronary arteries and confirmed the right AAF on aortogram (Fig. 3). She had an elevated left ventricular end diastolic pressure of $35 \mathrm{mmHg}$.

Additional laboratory work demonstrated a normal erythrocyte sedimentation rate, C-reactive protein, antinuclear antibody and extractable nuclear antigen panel, rapid plasma reagin, and FBN1 gene analysis.

The patient underwent a successful patch repair of the right AAF (Fig. 4). On follow-up, she was recovering well, with a postoperative TTE revealing normal right atrial and right ventricular pressures and no evidence of SVA or AAF. 


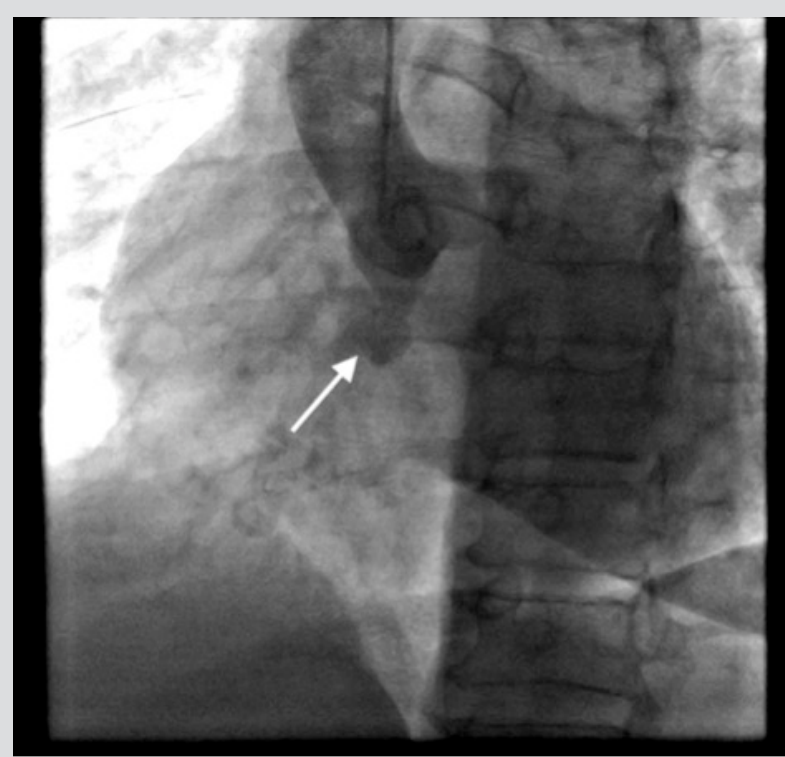

Figure 3. Aortogram showing an abnormal connection between an aorta and right atrium from ruptured SVA

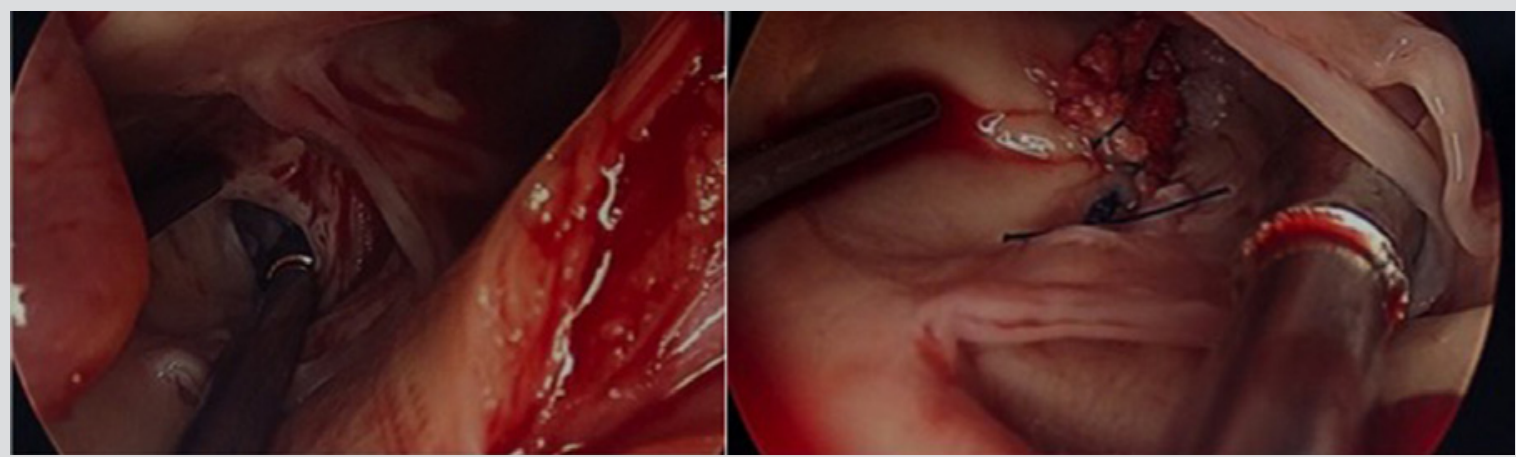

Figure 4. Intraoperative images demonstrating patch repair of the AAF

\section{DISCUSSION}

Unruptured SVAs are often clinically silent; however, large unruptured aneurysms may cause conduction abnormalities, such as complete heart block, myocardial ischemia from compression of coronary arteries, and right ventricular outflow tract obstruction causing acute right heart failure ${ }^{[8]}$. SVAs are most commonly diagnosed cardiac imaging like echocardiography and often found incidentally. SVA rupture is a devastating event that needs prompt recognition and surgical intervention ${ }^{[3]}$. The clinical symptoms and signs will depend on the size and speed of rupture and the cardiac chamber with which it communicates ${ }^{[10]}$. Physical examination will often reveal a new continuous machinery murmur ${ }^{[2]}$. One-half of these patients present with dyspnea, peripheral edema and chest pain worsening over several months ${ }^{[4,7]}$. Acute rupture is seen in one-third of patients producing dyspnea, chest pain and signs of acute heart failure, as observed in our case ${ }^{[7]}$. Since most right coronary sinus aneurysms and non-coronary sinus aneurysms rupture into the right side of the heart, progressive heart failure from left-to-right shunt and aneurysmal fistulization are observed ${ }^{[6]}$.

TTE is the preferred initial diagnostic study and identifies the presence and location of the SVA, with a sensitivity and specificity of $99 \%$ [10]. TTE also identifies aneurysmal rupture with a sensitivity of $97 \%$, but is limited in diagnosing a fistulous tract with a sensitivity of only $50 \%$ [10]. TEE has a higher sensitivity of close to $90 \%$ in recognizing fistulous tract formation ${ }^{[4]}$. Cardiac magnetic resonance imaging and CT are alternate modalities to confirm or better categorize echocardiographic findings ${ }^{[8]}$. In our patient, the fistula origin was unclear and the utilization of CT allowed for accurate diagnosis and surgical planning. Prior to these, other advanced imaging modalities such as cardiac catheterization with aortography were traditionally the gold standard for diagnosing SVA. Now it is more commonly performed as part of the preoperative evaluation of coronary anatomy and is reserved for cases where diagnosis is uncertain ${ }^{[2,4]}$. 
The mainstay of treatment of ruptured SVA is surgical repair ${ }^{[9]}$. Without surgical repair, the mean survival is 1 to 2 years in these patients ${ }^{[2]}$. The presence of left-to-right shunt, fistulization and acute congestive heart failure are indications for emergent surgical intervention, as was the case in our patient ${ }^{[2]}$. Patch repair is preferred by most cardiothoracic surgeons rather than simple closure as it minimizes aortic leaflet damage ${ }^{[9]}$. The long-term results of surgical repair of a ruptured SVA are excellent, with one large study reporting an $83.1 \%$ survival rate at 5 years ${ }^{[11]}$. Percutaneous transcatheter closure techniques are emerging for unruptured SVAs and selected patients with ruptured SVAs ${ }^{[12]}$.

\section{CONCLUSION}

It is imperative to be familiar and have an understanding of the possibility of a ruptured sinus of Valsalva aneurysm and aorto-atrial fistula as the etiology of acute heart failure. This mindfulness will enable timely diagnosis and appropriate treatment to improve patient outcomes.

\section{REFERENCES}

1. Liu F, Zhu Z, Ren J, Mu J. A rare cause of sudden dyspnea and unexpected death in adolescence: fistula from aortic sinus of Valsalva to right atrium. Int J Clin Exp Med 2014;7(9):2945-2947.

2. Moustafa S, Mookadam F, Cooper L, Adam G, Zehr K, Stulak J, et al. Sinus of Valsalva aneurysms - 47 years of a single center experience and systematic overview of published reports. Am J Cardiol 2007;99(8):1159-1164.

3. Liu S, Xu X, Ding X, Liu G, Zhao Z, Zhao X, et al. Comparison of immediate results and mid-term follow-up of surgical and percutaneous closure of ruptured sinus of Valsalva aneurysm. J Cardiol 2014;63(3):239-243.

4. Nabati M, Ghaffari R, Ghaemian A. Rupture of a noncoronary sinus of Valsalva aneurysm into the left atrium: a rare cause of acute pulmonary edema. Echocardiography 2013;30(10):e315-e318.

5. Shah BN, Livesey SA, Rakhit DJ. Aorto-atrial fistula in the absence of infective endocarditis: diagnosis by 2-and 3-dimensional transesophageal echocardiography. Tex Heart Inst J 2012;39(1):146-147.

6. Ananthasubramaniam K. Clinical and echocardiographic features of aorto-atrial fistulas. Cardiovasc Ultrasound 2005;3:1.

7. Arikan E, Karagöz A, Bayata S, Yilik L, Ünlüer EE. A rare cause of dyspnea: sudden rupture of aortic Valsalva sinus aneurysm. Case Rep Med $2013 ; 909302$.

8. Jugpal TS, Dixit R, Lohchab S, Garg A. Giant unruptured sinus of Valsalva aneurysm: an unusual cause of right heart failure. J Clin Imaging Sci 2015;5:64.

9. Vural KM, Şener E, Taşdemir O, Bayazit K. Approach to sinus of Valsalva aneurysms: a review of 53 cases. Eur J Cardiothorac Surg 2001;20(1):71-76.

10. Cheng TO, Yang YL, Xie MX, Wang XF, Dong NG, Su W, et al. Echocardiographic diagnosis of sinus of Valsalva aneurysm: a 17-year (1995-2012) experience of 212 surgically treated patients from one single medical center in China. Int J Cardiol 2014;173(1):33-39.

11. Takach TJ, Reul GJ, Duncan JM, Cooley DA, Livesay JJ, Ott DA, et al. Sinus of Valsalva aneurysm or fistula: management and outcome. Ann Thorac Surg 1999;68(5):1573-1577.

12. Tong S, Zhong L, Liu J, Yao Q, Guo Y, Shu M, et al. The immediate and follow-up results of transcatheter occlusion of the ruptured sinus of Valsalva aneurysm with duct occluder. J Invasive Cardiol 2014;26(2):55-59. 